\title{
Helsinki doctrine under review
}

$\mathrm{T}$ he World Medical Association will revise the Declaration of Helsinki before the core biomedical ethics document's 50th anniversary in 2014 , a process sure to spark intense debate over the use of placebos during clinical trials in poor countries, and guaranteed access to after-study care.

The review will seek consensus among physicians representing more than 100 countries on new draft guidelines to protect people participating in clinical trials. The association typically conducts such an update every five to six years.

But the next iteration will come as an increasing number of global bodies are creating their own research ethics guides, giving pharmaceutical companies and researchers several standards from which to choose. The European Commission, for instance, is currently revising its Clinical Trials Directive. The World Health Organization, meanwhile, has its own detailed regulations and guidance, the International Ethical Guidelines for Biomedical Research Involving Human Subjects, which was crafted in conjunction with the Council for International Organizations of Medical Sciences. Canada has its own guidelines: the Tri-Council Policy Statement: Ethical Conduct for Research Involving Humans.

That preponderance of competing ethical documents, all purporting to convey the highest possible ethical standards, leads some to question the Helsinki declaration's continued relevance.

"Ultimately, it's not an enforceable document - it doesn't hold the status of laws or regulations in any particular country," says Dr. Michael Carome, deputy director of Public Citizen's Health Research Group in Washington, D.C. "So while it's frequently cited as an important document that describes basic ethical principles for protecting human subjects in medical research, currently it doesn't bind any physician researcher in a legal way."

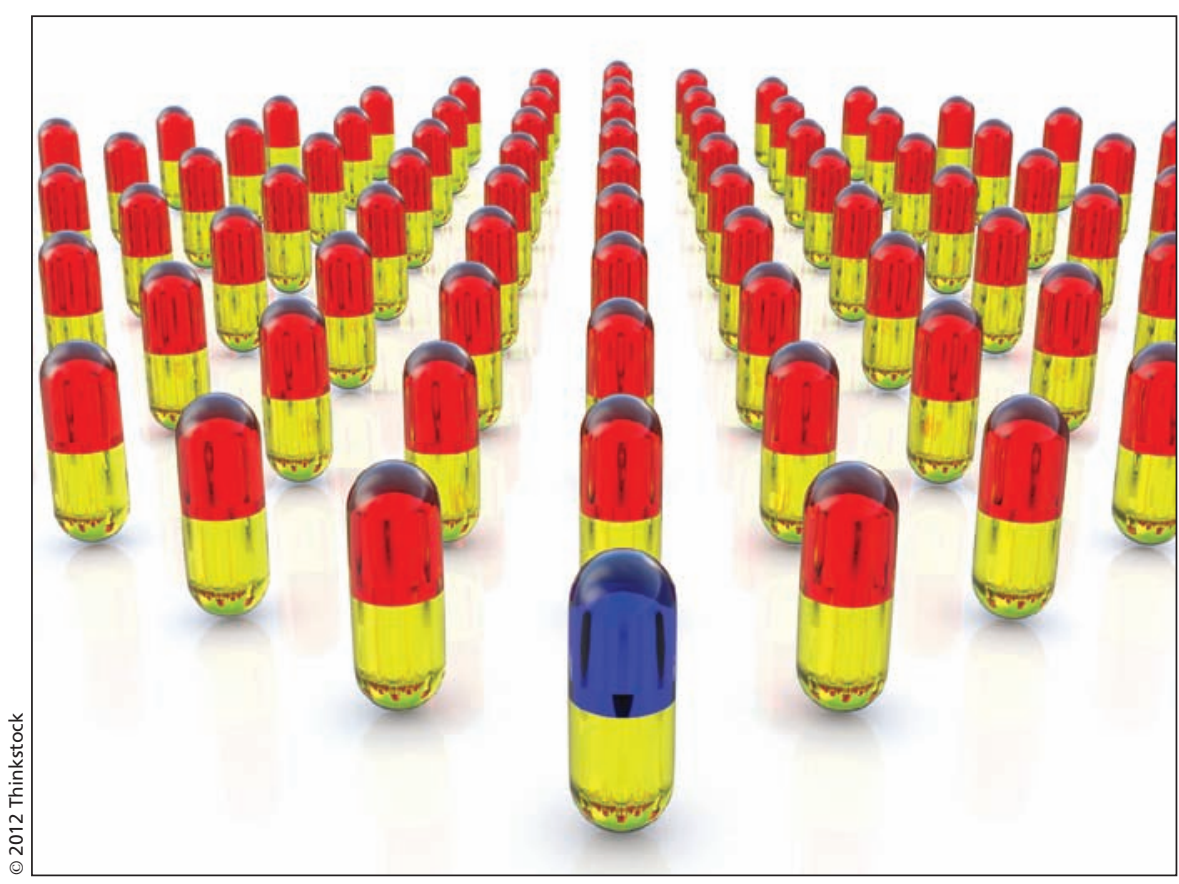

The use of placebos in resource-poor settings has long been a contentious issue.

"I would argue certainly that we need a more binding international instrument," says Trudo Lemmens, associate professor and the Scholl Chair in Health Law and Policy at the University of Toronto in Ontario. But he notes it is "a politically difficult issue" to draft and enforce such an agreement.

Helsinki's status is even weaker than that of any oft-violated United Nations treaty or resolution, Carome notes. In part, that's because if a country, pharmaceutical giant or funding agency disagrees with the Helsinki wording, it can adopt either higher or less stringent standards.

The United States Food and Drug Administration (FDA), for example, rejected the declaration's 2000 and later revisions, largely over the question of whether placebos should be allowed in clinical trials in resource-poor settings, says Lemmens. In 2006, the FDA announced it was adopting the Good Clinical Practice standards developed by the International Conference on Harmonisation of Technical Require- ments for Registration of Pharmaceuticals for Human Use as its ethics guide for clinical trials. Unlike the declaration, which was drafted by physicians for physicians, those standards are developed by regulators in Japan, the US and Europe, in conjunction with the pharmaceutical industry.

"Pharmaceutical companies ultimately look to see what are the regulations and laws they must comply with in whatever countries they are going to seek approval to market a particular product," says Carome. "To the extent that it's easier and perhaps less costly to conduct their research in settings ... that appear to have looser standards or less rigorous ethical processes, then we've seen a trend in which they have been moving more towards doing research in that setting."

Despite the variety of international guidelines, the declaration has value, argues Dr. Ramin Parsa-Parsi, chair of the association's Working Group on the Revision of the Declaration of Helsinki. Helsinki should be viewed as an umbrella for the other guidelines or 
regulations, he says, adding that it is the only ethical document produced "in a very international, global way, in a very democratic way."

"We don't have any influence by governments or by the industry," ParsaParsi adds. Although representatives from the International Federation of Pharmaceutical Manufacturers \& Associations are invited to weigh in on the Helsinki drafts, "we don't pay them and they don't pay us. They are just invited as guests to give their perspective." The association then uses all the information received to build its own opinion, which Parsa-Parsi believes results in a more neutral document.

The requirement for consensus among members dictates the length of the review process, which can take two to three years. Controversial topics are again expected to include whether companies are required to merely identify or to actually provide after-study care to trial participants; what constitutes "standard" care in a resourcepoor country or setting; and whether placebos should ever be used instead of standard care.

"What we're aiming for right now is a more nuanced approach than a complete ban on any type of research different than the research done in the Western context," says Dr. Jeff Blackmer, executive director of the Canadian Medical Association's Office of Ethics, Professionalism and International Affairs, and an ethics consultant to the Helsinki working group.

The challenge is to ensure the revision does not leave the door open for abuses or deprive people of potentially life-saving treatment in settings where they would not receive it but for a trial, Blackmer adds. "Our intent is to try and find a way, and a consensus, so that people in countries where there is a need for interventions for conditions that are important in those countries get access without being exploited."

The working group will also debate issues surrounding biobanks, working with vulnerable groups, the use of research ethics committees or boards, medical enhancement (everything from cosmetic surgery to cognitive improvements from so-called "medical foods'), and insurance compensation and protection.

There is a "heavy lobby" coming out of the United States and some other jurisdictions arguing that Helsinki and other research ethics guidelines should emphasize informed consent on the part of trial participants and considering whether risk is excessive when considering whether to use placebos, Lemmens says. Some researchers at the National Institutes of Health in the United States also advocate getting rid of standards that, for example, advocate against incentives for participation in trials, he adds.

"The question is, should you bend the research ethics guidelines more towards what's [already] going on, or should we stick to certain key principles and actually strengthen the review and enforcement of these guidelines?" asks Lemmens. He favours strengthening guidelines and having countries, including Canada, give them more force in legislation and regulation.

Carome would also like to tighten the declaration's language to limit the use of placebos where standards of care exist and to ensure trials are not conducted to develop treatments that will not be available in the countries where they are tested. - Laura Eggertson, CMAJ

CMAJ 2012. DOI:10.1503/cmaj.109-4295 